\title{
Zeeman Effect in Positronium Annihilation at Low Temperatures
}

\author{
William C. Sauder* and Richard D. Deslattes \\ Institute for Materials Research, National Bureau of Standards, Washington, D.C. 20234
}

(May 10, 1967)

\begin{abstract}
The annihilation of positronium is discussed as a means of obtaining a precise measurement of the Compton wavelength of the electron. In particular the spectral line shape of the annihilation radiation is calculated for positronium thermalized in helium gas cooled to the region of its critical point with an applied magnetic field sufficient to yield a three photon to two photon branching ratio of unity for the ${ }^{3} \mathrm{~S}_{0}$ component. Under such conditions the rapid thermalization of the positronium and the long half-life of two photon decay out of the predominantly triplet state make possible the production of rather narrow spectral distributions. An exact solution of the Zeeman effect in positronium is included. This study suggests that a wavelength determination beyond the one part per million level is attainable within the range of present technology.
\end{abstract}

Key Words: Annihilation radiation, Compton wavelength of the electron, positronium, quenching.

\section{Introduction}

X-ray data have been excluded from recent adjustments of the atomic constants $[1]^{1}$ since at their then current precision they would have carried typically about one fifth the weight assigned to other measurements. Simply improving the precision of the $x$-ray data by a factor of three would lead to equal weight. Recent improvements in the internal consistency of the $\mathrm{x}$-ray scale by Bearden et al. [2], permit a further sharpening by $x$-ray determinations of the constants to limits set by the uncertainty in the conversion factor between the $x$-ray wavelength scale and the defined length. Were these potentialities realized today, then the x-ray data would be significant in an adjustment of the fine structure constant and the proton moment [3].

The precision of $\mathrm{x}$-ray measurements of the atomic constants is alimited by the precision with which it is possible to define an x-ray wavelength scale. The scale, of course, depends on the precision with which it is possible to establish the wavelength of an individual $\mathrm{x}$-ray line. Ordinary $\mathrm{x}$-ray lines have full widths at half maximum upwards of some $300 \mathrm{ppm}$ (parts per million). We have no theoretical model for their shape and hence little hope of specifying the wavelength of any of their features (peak, centroid, or median) to better than $1 \mathrm{ppm}$. This problem has been studied by J. S. Thomsen and F. Y. Yap [4].

*Associate Professor of Physics, Virginia Military Institute, Lexington, Virginia 24450. Consultant to the Crystal Chemistry Section, Inorganic Materials, Division, National Bureau of Standards, Washington, D.C. 20234.

'Figures in brackets indicate the literature references at the end of this paper.
Beside the traditional $x$-ray determinations of the constants there are phenomena which either occur spontaneously or can be provoked to occur which are considerably sharper than natural $x$-ray lines. For example, there are nuclear transitions which have quite narrow profiles [5] and there are crystal lattices of extraordinary perfection [6] which occur as, so to speak, spontaneous phenomena. Other phenomena which are also quite sharp can be produced by experimental design. One of these, namely radiation from the annihilation of positronium as narrowed by thermalization in a magnetic field is the subject of the present paper.

\section{Positron-Electron Annihilation}

If a positron and an electron initially at rest annihilate with the emission of two photons, then by conservation of energy,

$$
2 \frac{h c}{\lambda_{A}}=2 m_{0} c^{2},
$$

since conservation of linear momentum implies that the wavelengths of the two photons are equal. Solving eq (1) for $\lambda$ yields the Compton wavelength:

$$
\lambda_{A}=\frac{h}{m_{0} c}
$$

where $m_{0}$ is the rest mass of either the electron or the positron. 
If, however, the positron-electron system has nonzero momentum at the instant of annihilation, the two photons in general will not have the same wavelength, so that spectroscopic observation of the radiation shows a broadened annihilation line. Examination of this process shows that the line widths resulting from the annihilation of free positrons with electrons in matter are much too great (typically in excess of 1000 ppm) to allow a precision (i.e., approx. 1 ppm) measurement.

If a flux of positrons is incident upon certain materials, a significant fraction of these incoming particles will combine with electrons in the substance to form hydrogen-like atoms of positronium [7]. The peak of the annihilation line is shifted because the righthand side of eq (1) is reduced by the binding energy of the positronium atom, while the width of the line is determined by the momentum of the positronium atom at the time of annihilation.

Positronium exists in either singlet (para-) or triplet (ortho-) states; the lifetime against annihilation of ortho-positronium is two orders of magnitude greater than that of para-positronium. The triplet lifetime is sufficiently great to allow an ortho-positronium atom to reach thermal equilibrium with its surroundings before annihilating. If this thermalization process is carried out in the presence of an external magnetic field, then an appreciable fraction of the long-lived positronium component will decay via the two photon channel because the field causes mixing of the singlet and triplet states. If the temperature of the surroundings is sufficiently low, then the resulting annihilation line will be narrow.

It is the purpose of this paper to examine in detail the line shape of the radiation produced in this process. In particular we shall consider the lifetime of the two photon annihilation of positronium in the presence of a magnetic field; combining this with appropriate expressions for the thermalization of the positronium and for the thermal distribution function of the surroundings yields an expression for the line shape.

\section{Zeeman Effect of Positronium}

The hyperfine separation between the singlet and triplet energy levels of positronium in its ground state is much greater than the corresponding splitting in the hydrogen atom for two reasons [8]. In the first place the dipole-dipole interaction between the electron and positron is proportional to the magnetic moment of the positron, and therefore is increased over the interaction in hydrogen by the ratio of the Bohr magneton to the nuclear magneton. Furthermore, the hyperfine splitting is increased by an amount that is approximately equal in magnitude to the dipole-dipole term by quantum electrodynamic effects involving virtual annihilation of the pair.

If $\mathfrak{S}_{0}$ represents the Hamiltonian (including the hyperfine interaction) of the positronium, then one can represent the stationary ground state as an eigenstate

$$
\mathfrak{S}_{0} u_{F m}=E_{F} u_{F m}
$$

where $F=0,1$ is the quantum number denoting the spin state of the atom, and $m$ is the quantum number associated with $z$-component of the spin angular momentum. The states $u_{F m}$ are eigenfunctions of the spin operators $\left(S_{e}+S_{p}\right)^{2}$ and $\left(S_{e z}+S_{p z}\right)$, where the subscripts $e$ and $p$ denote the electron and positron, respectively. These states, written in terms of the spin states of the individual particles, are

$$
\begin{gathered}
u_{11}=\Psi_{0} u_{e}(\uparrow) u_{p}(\uparrow) \\
u_{10}=\frac{\Psi_{0}}{\sqrt{2}}\left[u_{e}(\uparrow) u_{p}(\downarrow)+u_{e}(\downarrow) u_{p}(\uparrow)\right] \\
u_{1-1}=\Psi_{0} u_{e}(\downarrow) u_{p}(\downarrow) \\
u_{00}=\frac{\Psi_{0}}{\sqrt{2}}\left[u_{e}(\uparrow) u_{p}(\downarrow)-u_{e}(\downarrow) u_{p}(\uparrow)\right]
\end{gathered}
$$$$
\text { and }
$$

where $\Psi_{0}$ is the hydrogenic ground state wave function which results if spin is neglected. The hfs separation has been evaluated to be

$$
\Delta E=E_{1}-E_{0}=8 \times 10^{-4} \mathrm{eV} .
$$

If an external magnetic field $H$ is applied to the positronium atom, then the Hamiltonian becomes

$$
\mathfrak{S c}=\mathfrak{S}_{0}+\mathfrak{S}_{H}
$$

where, in terms of magnetic moment operators $\mu$ for the positron and electron,

$$
\mathfrak{S C}_{H}=-\left(\boldsymbol{\mu}_{e}+\boldsymbol{\mu}_{p}\right) \cdot \mathbf{H} \text {. }
$$

Since the magnetic moments of the electron and positron are equal (approximately) in magnitude to the Bohr magneton but opposite in direction with respect to the spin vectors, and since their $g$ factors are equal, then $\mathfrak{S}_{H}$ can be written in terms of Pauli spin matrices $\sigma_{z}$ as

$$
\mathfrak{S}_{H}=\frac{\mu_{0} g H}{2}\left(\sigma_{e z}-\sigma_{p z}\right) \text {. }
$$

Some treatments [9] of the Zeeman effect in positronium have employed perturbation theory; however, an exact solution is readily obtained by well-known methods [10].

One can write down the Hamiltonian matrix by employing the fact that $\sigma_{z} u(\uparrow)=u(\uparrow)$ and $\sigma_{z} u(\downarrow)$ 
$=-u(\downarrow)$; the operation of $\mathfrak{S}_{H}$ in eq (7) on the wave functions in eq (4a-d) yields

and

$$
\begin{aligned}
& \mathfrak{S}_{H}\left|u_{11}=\mathfrak{S}_{H}\right| u_{1-1}>=0 \\
& \mathfrak{S}_{H}\left|u_{10}>=\mu_{0} g H\right| u_{00}>
\end{aligned}
$$

$$
\mathfrak{S}_{H}\left|u_{00}>=\mu_{0} g H\right| u_{10}>\text {. }
$$

As a result, the Hamiltonian matrix including the magnetic interaction is

$$
\langle\tilde{S}\rangle=\left(\begin{array}{llll}
E_{1} & 0 & 0 & 0 \\
0 & E_{1} & 0 & \mu_{0} g H \\
0 & 0 & E_{1} & 0 \\
0 & \mu_{0} g H & 0 & E_{0}
\end{array}\right)
$$

The secular equation for $\langle\mathfrak{S}\rangle$ is

$$
\left(E_{1}-\lambda\right)^{2}\left\{\left(E_{1}-\lambda\right)\left(E_{0}-\lambda\right)-\left(\mu_{0} g H\right)^{2}\right\}=0
$$

with roots

$$
\lambda=E_{1}, E_{1}, \bar{E} \pm \frac{1}{2} \Delta E \sqrt{1+x^{2}}
$$

where

and

$$
\begin{aligned}
& \bar{E}=\frac{1}{2}\left(E_{1}+E_{0}\right) \\
& x=\frac{\mu_{0} g H}{\Delta E / 2} .
\end{aligned}
$$

Thus the diagonalized matrix is

$$
\left\langle\mathfrak{S}_{\mathrm{C}}\right\rangle_{\mathrm{diag}}=\left(\begin{array}{cccc}
\mathrm{E}_{1} & 0 & 0 & 0 \\
0 & \bar{E}+\frac{\Delta E}{2} \sqrt{1+x^{2}} & 0 & 0 \\
0 & 0 & E_{1} & 0 \\
0 & 0 & 0 & \bar{E}-\frac{\Delta E}{2} \sqrt{1+x^{2}}
\end{array}\right)
$$

Note that the degeneracy between the triplet $m= \pm 1$ states is not removed by $\mathfrak{S}_{H}$. Furthermore, the energy of these states is not affected by the field, since these states have zero net magnetic moment because the positron and electron are oppositely charged particles. The lack of a linear Zeeman effect, and the existence of a quadratic effect in the $m=0$ state can be described qualitatively as follows. The triplet $m=0$ state as mentioned above has no magnetic moment and is not affected in first order. The singlet state, though it possesses a magnetic moment, is a zero spin state; since there is no axis of quantization, the atom "tumbles" in the field. Both states do exhibit an energy shift because the applied field induces a preferential alinement of the individual particle magnetic moments as is indicated by the $\mathrm{H}^{2}$ variation (in the limit, this induced alinement becomes the Paschen-Back effect).

The wave functions for the perturbed positronium atom can now be determined by carrying out the matrix multiplication

$$
\langle S\rangle\langle\mathfrak{S}\rangle=\langle\mathfrak{S}\rangle_{\operatorname{diag}}\langle S\rangle
$$

where $\langle S\rangle$ is the transformation matrix that diagonalizes $\langle\mathfrak{S}\rangle$, and employing the fact that $\langle S\rangle$ must be unitary. This operation leads to the following wave functions:

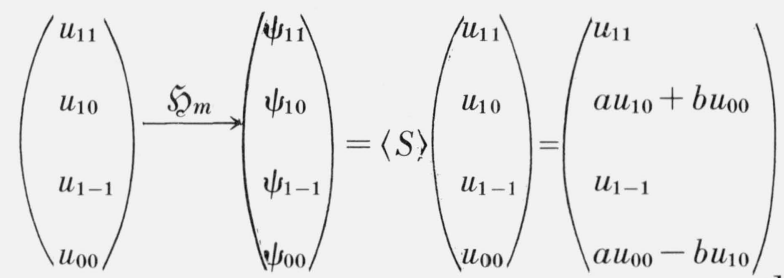

where

$$
a=\frac{1}{\sqrt{2}}\left(1+\frac{1}{\sqrt{1+x^{2}}}\right)^{1 / 2}
$$

and

$$
b=\frac{1}{\sqrt{2}}\left(1-\frac{1}{\sqrt{1+x^{2}}}\right)^{1 / 2}
$$

Equation (14) shows that the application of a magnetic field mixes the states $u_{10}$ and $u_{00}$. Thus $\psi_{10}$ and $\psi_{00}$ each decay by both the two- and three-photon channels.

If the annihilation rate of the state $u_{10}$ is $\gamma_{T}$ and that for the state $u_{00}$ is $\gamma_{s}$, then the mean annihilation rate for the mixed state $\psi_{10}$ is

$$
\gamma_{10}=a^{2} \gamma_{T}+b^{2} \gamma_{s}
$$

Substitution of eq (15) into the above yields

$$
\gamma_{10}=\left(\frac{\gamma_{T}+\gamma_{s}}{2}\right)+\frac{1}{\sqrt{1+x^{2}}}\left(\frac{\gamma_{T}-\gamma_{s}}{2}\right)
$$

The branching ratio for the $\psi_{10}$ state (i.e., the ratio of the number of two photon decays to three photon decays) is

$$
B_{10}=\left(\frac{b}{a}\right)^{2} \frac{\gamma_{s}}{\gamma_{T}}=\frac{\left(2+x^{2}\right)-2 \sqrt{1+x^{2}}}{x^{2}} \frac{\gamma_{s}}{\gamma_{T}} .
$$


In figure 1 are plotted eqs (17) and (18) as functions of the applied field $H$ up to $2177 \mathrm{G}$, at which point $B_{10}$ equals unity.

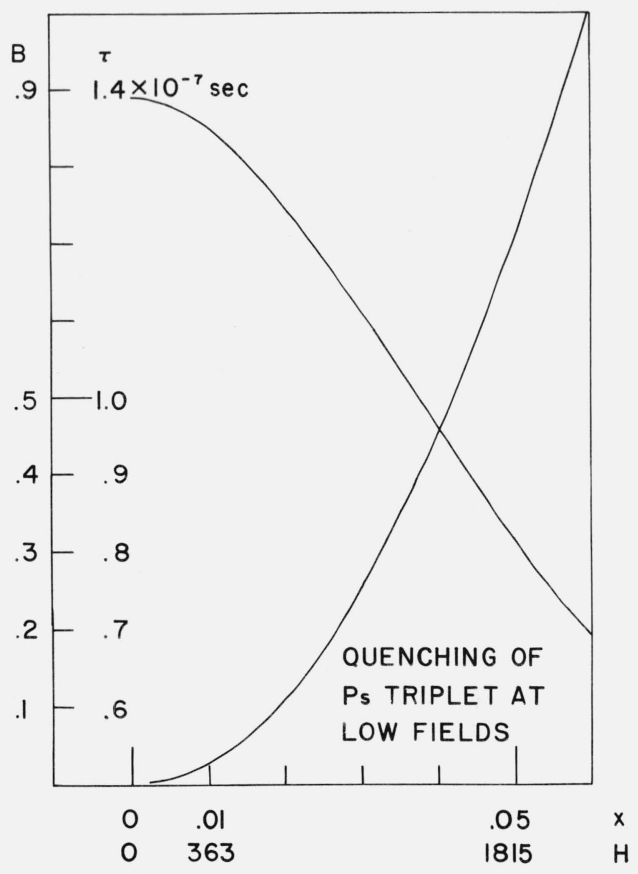

FIGURE 1. Quenching of triplet positronium at low fields.

\section{Line Profile for Positronium in a Mono- energetic Moderator}

In this section we shall consider the case in which a flux of positronium atoms is incident in a moderating medium possessing a single thermal energy. We must first relate the energy separation of the two photons to their emission directions. Figure $2 \mathrm{a}$ is a representation of the annihilation of a positron atom with linear momentum p into photons $h \nu_{1}$ and $h \nu_{2}$ which are emitted at an angle $\epsilon$ with respect to each other. The photon $h \nu_{1}$ is emitted at an angle $\theta$ with respect to the direction of the incident positronium atom. From the momentum triangle of figure $2 b$ one can write

$$
p_{2}^{2}-p_{1}^{2}=p^{2}-2 p p_{1} \cos \theta .
$$

Letting $h \nu_{1}$ be the photon of greater energy, then one can write

$$
h \nu_{1}=\frac{1}{2} E_{0}+\delta
$$

and

$$
h \nu_{2}=\frac{1}{2} E_{0}-\delta,
$$

where $E_{0}$ is the total energy of the positronium atom prior to annihilation. Thus multiplying eq (19) by $c^{2}$

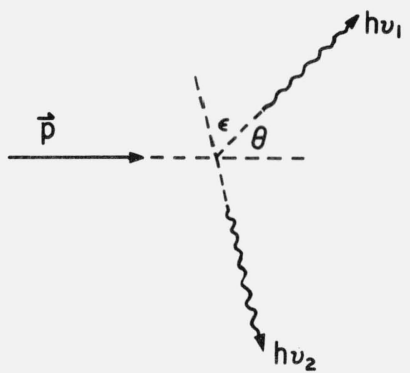

(a)

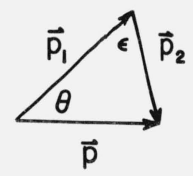

(b)

FIGURE 2. (a) Two-photon annihilation of positronium in motion. (b) Momentum triangle for the process $(a)$.

and subsequently substituting eqs (20) into the result leads to

$$
\delta=-\frac{p c}{2}\left\{\frac{p c}{E_{0}}-\left(1+\frac{2 \delta}{E_{0}}\right) \cos \theta\right\}
$$

If the positronium atom is nearly in thermal equilibrium with its surroundings at annihilation, then

$$
\frac{p c}{E_{0}} \ll 1
$$

and

$$
\frac{2 \delta}{E_{0}} \ll 1 ;
$$

as a result, eq (21) can be written

$$
\delta \simeq \frac{p c}{2} \cos \theta=\delta_{\max } \cos \theta .
$$

It has been shown by Heitler [11] that the emission of annihilation photons is spherically symmetric in the limit $\mathbf{p}=0$; thus the distribution will be very nearly isotropic in the case at hand-that of thermalized positronium. The intensity distribution as a function 
of $\theta$ will thus be proportional to the solid angle subtended at the annihilation site: ${ }^{3}$

$$
I_{\theta} d \theta=\frac{1}{2} \sin \theta d \theta, \theta<\frac{\pi}{2}
$$

Then the intensity distribution for $\delta$ can be written

$$
I_{\delta} d \delta=I_{\theta} d \theta=I_{\theta} \frac{d \theta}{d \delta} d \delta, \delta<\delta_{\max }
$$

The quantity $d \theta / d \delta$ can be evaluated from eq (22) so that the distribution function $I_{\delta} d \delta$ in eq (24) becomes

$$
I_{\delta} d \delta=\frac{d \delta}{p c}, \delta<\delta_{\max } .
$$

Thus the annihilation of a positronium atom traveling with momentum $\mathbf{p}$ is equally likely to produce photons in the range from $E_{0} / 2$ up to $E_{0} / 2+p c / 2$.

It is now possible to determine the annihilation line shape from a positronium flux in a monoenergetic moderator. The number of $P s$ atoms annihilating during the time interval $(t, t+d t)$ is

$$
N_{t} d t=\gamma_{10} N_{0} e^{-\gamma_{10} t} d t .
$$

Annihilations during this time interval contribute an increase of intensity $d I_{\delta}$ which is uniform for all $\delta$ from 0 up to $\delta_{\max }$, according to eq (25). Since the area under the distribution curve is equal to the number of atoms annihilating, then

$$
N_{t} d t=\delta_{\max } d I_{\delta}
$$

We can determine the total intensity distribution by adding up all the $d I_{\delta}$ 's from $t=0$ up to $t_{\delta}$ such that $\delta=\delta_{\max }$ :

$$
I_{\delta}=\int_{0}^{t_{\delta}} \frac{N_{t} d t}{p c}
$$

The momentum $p=\sqrt{2 m E}$ is a function of the time; this thermalization function has been found [12] to be

$$
E=E_{m} \operatorname{coth}^{2}(\beta+\alpha t)
$$

where $E_{m}$ is the moderator energy; $\beta$ depends upon the energy of the positronium atoms at $t=0$, and $\alpha$ depends upon the properties of the moderator. Defining

$$
u=\beta+\alpha t
$$

eq (28) can be written as

$$
I_{\delta}=K \int_{0}^{t_{\delta}} e^{-\frac{\gamma_{10} u}{d}} \tanh u d u .
$$

${ }^{3}$ In reality $\theta$ does not quite reach $\pi / 2$; this is merely an indication of the approximation of spherical symmetry, and will not affect the results significantly.
This integral can be approximated in the case for which $\gamma_{10} / \alpha$ is small. The details of this calculation appear in the appendix. The resulting distribution function is

$$
\begin{aligned}
I_{\delta} & =I_{0}\left\{1-\left[1-\left(\frac{\delta_{T}}{\delta}\right)^{2}\right]^{\left.\frac{\gamma_{10}}{2 \alpha}\right\}} \quad \delta>\delta_{T}\right. \\
& =I_{0} \quad 0<\delta<\delta_{T}
\end{aligned}
$$

where $\delta_{T}$ is the maximum energy shift for the annihilation of a completely thermalized positronium atom.

In the case of two-photon annihilation of the $\psi_{10}$ component of positronium using helium at its critical point $\left(T_{c}=5.2^{\circ} \mathrm{K}, p=2.3 \mathrm{~atm}\right)$ as a moderator and an applied magnetic field of $2177 \mathrm{G}$ (so that $B_{10}=1$ ), the quantity $\gamma_{10} / \alpha$ is quite small:

$$
\left.\frac{\gamma_{10}}{\alpha}\right|_{\substack{\mathrm{He} \mathrm{crit} \\ 2177 \mathrm{G}}}=8.5 \times 10^{-3} .
$$

As a result the function $I_{\delta}(\mathrm{eq}(31)$ ) falls very rapidly for $\delta>\delta_{T}$, so that this leads to a distribution function that is nearly rectangular; viz, at $\delta / \delta_{T}=1, I=I_{0}$, while at $\delta / \delta_{T}=1.001, I=I_{0} \times 10^{-3}$.

The physical interpretation of this result is fairly simple. Since $\gamma_{10}$ is the two photon annihilation rate of the long-lived component of positronium, then $\gamma_{10}^{-1}$ is the mean lifetime of the atom; similarly, $\alpha^{-1}$ represents the characteristic time required for the $P s$ atom to thermalize with its surroundings. Thus the ratio $\gamma_{10 / \alpha}$ is just the ratio of thermalization time to mean life. When this ratio is small, then thermalization usually is completed long before the positronium atom decays. Since the calculations of this section concern the somewhat artificial case of a monoenergetic moderator, the resulting rectangular. distribution in the preceding paragraph indicates that the vast majority of the positronium atoms decay after they have become fully thermalized, so that the resulting intensity distribution is simply the characteristic rectangular distribution arising from monoenergetic positronium.

\section{Line Profile for Thermalized Positronium}

Let us now consider annihilation in a moderator at temperature $T$ characterized bv a Maxwell distribution of velocities:

$$
N_{v} d v=A v^{2} e^{-\frac{m v^{2}}{2 k T}} d v .
$$

If the thermalization time $\alpha^{-1}$ is much less than the mean life $\gamma_{10}^{-1}$, then most $P s$ atoms are in thermal equilibrium with the moderator at the time of annihilation. As a result there will be a Maxwell distribution of positronium atoms; atoms with velocities 
in the interval $(v, v+d v)$ will produce a rectangular distribution according to eq (31), so that

$$
\delta d I=N_{v} d v
$$

where $\delta$ is the width of the distribution and is the maximum energy shift for photons produced in the decay of a $P s$ atom moving with velocity $v$. If one makes the substitution $\delta=1 / 2 m v c$, eq (33) can be integrated to yield the intensity distribution of annihilation photons:

$$
\begin{gathered}
I=\int_{\delta / m c}^{\infty} \frac{2 A}{m v c} e^{-\frac{m v^{2}}{2 k T}} d v \\
=K e^{-\frac{m}{2 k T}\left(\frac{\delta}{m c}\right)^{2}} .
\end{gathered}
$$

The quantity $\delta$ can be expressed in terms of the positronium kinetic energy as

$$
\delta=c \sqrt{2 m E} .
$$

Denoting as $\delta_{T}$ that value of $\delta$ corresponding to the mean thermal energy at $T$, then one can write that

$$
\delta_{T}=c \sqrt{2 m\left(\frac{3}{2} k T\right)}=\sqrt{\left(\frac{3}{2} k T\right) E_{0}} .
$$

Since $E_{0} \simeq 2 m_{0} c^{2}$, then eq (34) becomes

$$
I=K e^{-\frac{3}{2}\left(\frac{\delta}{\delta_{r}}\right)^{2}}
$$

The line profile resulting from the annihilation process is a Gaussian with a half width at half intensity of

$$
\delta_{1 / 2}=\delta_{T} \sqrt{\frac{2}{3} \ln 2}=0.68 \delta_{T}
$$

\section{Summary}

It has been demonstrated that if a moderator is such that the thermalization time of positronium is much less than the mean life against annihilation, the two photon annihilation of positronium in this moderator at temperature $T$ results in an annihilation line that is a Gaussian, the width of which depends upon $T$. These conditions are fulfilled for helium near its critical point for positronium lifetimes of the order of magnitude of the triplet state lifetime. Since triplet positronium decays with the emission of three photons, whereas a determination of the Compton wavelength is feasible only in the case of two photon decay, one must carry out such an experiment in the presence of a magnetic field so that the mixing of singlet and triplet states give rise to a long-lived two photon process. It has been shown that for fields of approximately $2 \mathrm{kG}$, one half of the long-lived decays will occur via the two photon channel with a life time sufficiently great to fulfill the conditions for the Gaussian line shape described above. It would thus appear possible to carry out an annihilation experiment in which the line width is sufficiently narrow to allow a $1 \mathrm{ppm}$ determination of $\lambda_{A}$.

\section{Appendix}

In order to evaluate the integral of eq (30),

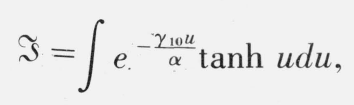

for the case in which $\epsilon=\left(\gamma_{10} / \alpha\right) \ll 1$, we make the substitution

$$
\eta=\ln \cosh u \text {. }
$$

The expression tanh $u d u$ can be expressed in terms of $\eta$ :

$$
\tanh u d u=\frac{d(\cosh u)}{\cosh u}=d(\ln \cosh u)=d \eta .
$$

From (A-2) we can write

$$
e^{\eta}=\cosh u
$$

which leads to

$$
u=\cosh ^{-1} e^{\eta}
$$

and therefore $(\mathrm{A}-1)$ becomes

$$
\Im=\int e^{-\epsilon \cosh ^{-1} e^{\eta}} d \eta \text {. }
$$

Employing an identity for $\cosh ^{-1}$ yields

$$
\begin{aligned}
\cosh ^{-1} e^{\eta}=\ln \left(e^{\eta}+\sqrt{e^{2 \eta-1}}\right) & \\
& =\eta+\ln \left(1+\sqrt{1-e^{-2 \eta}}\right)
\end{aligned}
$$

and therefore

$$
e^{-\epsilon \cosh -1} e^{\eta}=e^{-\epsilon \eta}\left(1+\sqrt{1-e^{-2 \eta}}\right)-\epsilon .
$$

Since $\epsilon$ is small, eq $(A-8)$ can be expanded:

$$
\begin{array}{r}
\left(1+\sqrt{1-e^{-2 \eta}}\right)-\epsilon=1-\epsilon \ln \left(1-\sqrt{1-e^{-2 \eta}}\right) \\
+\frac{\epsilon^{2}}{2} \ln ^{2}\left(1+\sqrt{1-e^{-2 \eta}}\right)-\ldots
\end{array}
$$

But note that

$\ln \left(1+\sqrt{1-e^{-2 \eta}}\right)=\sum_{s=1}^{x_{i}} \frac{(-1)^{s+1}}{s}\left(1-e^{-2 \eta}\right)^{s / 2}$

and that one can write

$$
\left(1+\sqrt{1-e^{-2 \eta}}\right)-e=1+\Phi \epsilon
$$


in which

$$
|\Phi| \epsilon \leqslant \epsilon \ln \left(1+\sqrt{1-e^{-2 \eta}}\right) \leqslant \epsilon\left(1-e^{-2 \eta}\right)^{1 / 2} .
$$

Now the integral $(A-6)$ becomes

$$
\Im=\int e^{-\epsilon \eta}(1+\Phi \epsilon) d \eta=-(1+\bar{\Phi} \epsilon) \frac{e^{-\epsilon \prime \prime}}{\epsilon}
$$

where

$$
\bar{\Phi}=\int \Phi e^{-\epsilon \eta} d \eta / \int e^{-\epsilon \eta} d \eta, \quad 0<\bar{\Phi}<1
$$

Rewriting (A-12) in terms of $u$ yields eq (31) in section 3 .

\section{References}

[1] E. R. Cohen and J. W. M. DuMond, Rev. Mod. Phys. 37, 537 (1965).
[2] J. A. Bearden, A. Henins, J. G. Marzolf, W. C. Sauder, and J. S. Thomsen, Phys. Rev. 135, A899 (1964).

[3] This situation is rapidly changing, see, e.g., W. H. Parker, B. N. Taylor and D. N. Langenberg, Phys. Rev. Letters, 18, 287 (1967).

[4] J. S. Thomsen and F. Y. Yap, to be published.

[5] J. A. Bearden, Phys. Rev. 137, B181 (1965).

[6] This is most graphically demonstrated by U. Bonse and M. Hart, Appl. Phys. Letters 7, 99 (1965).

[7] M. Deutsch, Prog. Nuc. Phys. 3, 131 (1953); P. R. Wallace, Solid State Physics, 10, 1 (Academic Press, New York, 1960).

[8] V. W. Hughes, S. Marder and C. S. Wu, Phys. Rev. 106, 934 (1957).

[9] O. Halpern, Phys. Rev. 94, 904 (1954); Hughes et al., ibid.

[10] E. V. Condon and G. H. Shortley, The Theory of Atomic Spectra, p. 152 (Cambridge University Press, New York, 1953).

[11] W. Heitler, The Quantum Theory of Radiation, 3d ed. (Oxford University Press, New York, 1954).

[12] William C. Sauder, J. Res. NBS 72A (Phys. and Chem.) No. 1 (1968).

(Paper 71A5-464) 